Model-based estimation of the frequency tuning of the inner-hair-cell stereocilia from neural tuning curves

Alessandro Altoè and Ville PulkkiSarah Verhulst

Citation: The Journal of the Acoustical Society of America 141, 4438 (2017); doi: 10.1121/1.4985193

View online: http://dx.doi.org/10.1121/1.4985193

View Table of Contents: http://asa.scitation.org/toc/jas/141/6

Published by the Acoustical Society of America 


\title{
Model-based estimation of the frequency tuning of the inner-hair- cell stereocilia from neural tuning curves
}

\author{
Alessandro Altoè ${ }^{\text {a) }}$ and Ville Pulkki \\ Department of Signal Processing and Acoustics, School of Electrical Engineering, Aalto University, \\ P.O. Box 13000, FI-00076 Aalto, Finland \\ Sarah Verhulst \\ Department of Information Technology, Ghent University, Technologiepark 15, 9052 Zwijnaarde, Belgium
}

(Received 29 November 2016; revised 19 May 2017; accepted 24 May 2017; published online 15 June 2017)

\begin{abstract}
This study proposes that the frequency tuning of the inner-hair-cell (IHC) stereocilia in the intact organ of Corti can be derived from the responses of the auditory fibers (AFs) using computational tools. The frequency-dependent relationship between the AF threshold and the amplitude of the stereocilia vibration is estimated using a model of the IHC-mediated mechanical to neural transduction. Depending on the response properties of the considered AF, the amplitude of stereocilia deflection required to drive the simulated AF above threshold is 1.4 to $9.2 \mathrm{~dB}$ smaller at low frequencies $(\leq 500 \mathrm{~Hz})$ than at high frequencies $(\geq 4 \mathrm{kHz})$. The estimated frequency-dependent relationship between ciliary deflection and neural threshold is employed to derive constant-stereociliadeflection contours from previously published AF recordings from the chinchilla cochlea. This analysis shows that the transduction process partially accounts for the observed differences between the tuning of the basilar membrane and that of the AFs. (C) 2017 Acoustical Society of America.

[http://dx.doi.org/10.1121/1.4985193]
\end{abstract}

$[\mathrm{PXJ}]$

Pages: 4438-4451

\section{INTRODUCTION}

The inner hair cells (IHCs) of the mammalian cochlea are the major sensory receptors of the auditory system, and they sense the vibrations of the organ of Corti (OC) through their stereocilia. The stereocilia base is firmly attached to the reticular lamina (RL) and their tip is free-standing in the fluid-filled space between the RL and the tectorial membrane (TM) (Lim, 1980). Because of the free-standing nature of the IHC stereocilia, their motion is determined by the fluidflow in their surroundings, which is determined by the nonlinear interaction of the different modes of vibrations of the organ of Corti (Guinan, 2012). Consequently, the mechanisms responsible for the vibration of the IHC ciliary bundles are poorly characterized at the present time.

While sound-induced vibration of the cochlea is currently well established in vivo for the basilar membrane (BM), little is known about the vibrations of the OC structures in proximity of the IHC stereocilia. Recently, the development of optical coherence tomography methods has made it possible to record the vibrations of the RL (Chen et al., 2011; Ramamoorthy et al., 2014; Ren et al., 2016a; Ren et al., 2016b) and the TM (Lee et al., 2015) in vivo. However, the available experimental data for these structures are currently insufficient to draw a precise picture of how the OC vibrates and how these vibrations couple to those of the stereocilia.

While physical modeling of the cochlear micro-mechanics (e.g., Steele and Puria, 2005; Steele et al., 2009) offers a

${ }^{\text {a)} E l e c t r o n i c ~ m a i l: ~ a l e s s a n d r o . a l t o e @ a a l t o . f i ~}$ fundamental tool to understand how the vibrations of the OC couple to those of the stereocilia, this approach requires the estimation of numerous physical properties of the cochlea, which are not easily determined in sensitive preparations.

This study proposes a complementary but different approach to characterizing the vibrations of the IHC stereocilia, which relies on estimating the mechanical drive to the IHC from the activity of the afferent auditory fibers (AFs) via computer simulations. Specifically, the frequency tuning of the IHC stereocilia is derived from the frequency tuning of the AFs in the mammalian base. The idea behind this approach is that the relationship between vibrations of the IHC stereocilia and the activity of the afferent AFs is primarily determined by the electrochemical properties of the IHC, which have been well characterized in the last two decades. The kinetics of the most prominent currents in the IHC have been documented in mammalian cells using in vitro preparations, which mimic the physiological chemical environment of the IHC (Kros et al., 1998; Marcotti et al., 2004; Jia et al., 2007; Johnson and Marcotti, 2008; Johnson et al., 2011; Johnson, 2015). Additionally, it has been shown that the whole-cell current flowing through the $\mathrm{Ca}_{\mathrm{V}} 1.3$ channels and the rate of exocytosis of neurotransmitter are approximately linearly related in high-frequency IHCs (Brandt et al., 2005; Johnson et al., 2005; Goutman and Glowatzki, 2007; Johnson et al., 2008; Wong et al., 2014) and that a quantal release of neurotransmitter is sufficient to trigger an action potential in the postsynaptic fiber (Siegel, 1992; Rutherford et al., 2012).

This knowledge about neural transduction can be employed to deduce the mechanical drive to the IHC from the activity of the AFs for specific cases using a computational- 
TABLE I. Model parameters.

\begin{tabular}{|c|c|c|}
\hline Parameter & Value & Meaning \\
\hline$C_{\mathrm{m}}$ & $12.5 \mathrm{pF}$ & IHC capacitance \\
\hline $\mathrm{EP}$ & $90 \mathrm{mV}$ & endocochlear potential \\
\hline \multicolumn{3}{|c|}{ MET channels } \\
\hline$G_{\max }$ & $30 \mathrm{nS}$ & max. conductance \\
\hline$x_{0}$ & $35 \mathrm{~nm}$ & offset \\
\hline$s$ & $16 \mathrm{~nm}$ & sensitivity \\
\hline$\tau_{\text {MET }}$ & $50 \mu \mathrm{s}$ & activation time constant \\
\hline \multicolumn{3}{|c|}{ Fast (f) and slow (s) $\mathrm{K}^{+}$channels } \\
\hline$G_{\mathrm{K}, \mathrm{f} / \mathrm{s}}$ & $230 \mathrm{nS}$ & max. conductance \\
\hline$V_{0.5}$ & $-31 \mathrm{mV}$ & half activation potential \\
\hline$s$ & $10.5 \mathrm{mV}$ & sensitivity \\
\hline$E_{\mathrm{K}, \mathrm{f}}$ & $-71 \mathrm{mV}$ & reversal potential \\
\hline$E_{\mathrm{K}, \mathrm{s}}$ & $-78 \mathrm{mV}$ & reversal potential \\
\hline$\tau_{\mathrm{K}, \mathrm{f}}$ & $0.3 \mathrm{~ms}$ & activation time constant \\
\hline$\tau_{\mathrm{K}, \mathrm{s}}$ & $8 \mathrm{~ms}$ & activation time constant \\
\hline \multicolumn{3}{|c|}{$\mathrm{Ca}^{2+}$ channels } \\
\hline$G_{\mathrm{Ca}}$ & $4.1 \mathrm{nS}$ & max. conductance \\
\hline$V_{0.5}$ & $-25 \mathrm{mV}$ & half activation potential \\
\hline$s$ & $7.5 \mathrm{mV}$ & sensitivity \\
\hline$\tau_{\mathrm{Ca}}$ & $0.2 \mathrm{~ms}$ & activation time constant \\
\hline$E_{\mathrm{Ca}}$ & $45 \mathrm{mV}$ & reversal potential \\
\hline
\end{tabular}

model approach. The proposed approach has several advantages over vibrometry. First, a large amount of AF data is available for modeling, whereas imaging the tiny vibrations of the stereocilia in sensitive preparations is currently not possible. Second, recordings from the AFs require simpler and less invasive techniques than those from the OC and hence they are less affected by recording artifacts.

The proposed method is best suited for studying levels of neural excitation near the neural threshold, i.e., for small increases of the average discharge rate of the considered AFs over its spontaneous rate. This consideration allows for the omission of some of the aspects of neural transduction that are not sufficiently characterized (e.g., synaptic depression), as they do not play a major role in estimating the threshold levels of the AFs using standard procedures.

\section{METHODS}

In the following, the model of mechanical-to-neural transduction employed to estimate the relationship between neural and stereocilia frequency tuning is presented. A summary of the model parameter values is given in Table I. The model is mostly based on in vitro measurements of the IHC and uses standard equations to describe the kinetics of the different ion channels. To keep the number of parameters as small as possible, the mathematical description of particular aspects of transduction is simplified in cases where this does not significantly affect the outcomes of the present analysis. An overview of the model is presented in Fig. 1.

\section{A. Mechanoelectric transduction}

\section{IHC membrane potential}

The present model of the IHC membrane potential (Fig. 1) is similar to the previously published biophysical models by Zeddies and Siegel (2004) and Lopez-Poveda and Eustaquio-Martín (2006). The membrane potential is mostly determined by the interplay between the mechanoelectric transduction (MET) current $I_{\mathrm{MET}}$ and the outward voltagedependent fast and slow activating $\mathrm{K}^{+}$currents $\left(I_{\mathrm{K}, \mathrm{f} / \mathrm{s}}\right)$. Solving the circuit in Fig. 1, the membrane potential $V_{\mathrm{m}}$ is determined by

$$
C_{\mathrm{m}} \frac{\mathrm{d} V_{\mathrm{m}}}{\mathrm{d} t}+I_{\mathrm{MET}}+I_{\mathrm{K}, \mathrm{f}}+I_{\mathrm{K}, \mathrm{s}}=0
$$

where $V_{\mathrm{m}}$ is the membrane potential and $C_{\mathrm{m}}$ the IHC capacitance.

We adopt $C_{\mathrm{m}}=12.5 \mathrm{pF}$ based on the recordings from IHCs of mature gerbils by Johnson et al. (2011). This value agrees well with that found for other rodents (Kros and Crawford, 1990; Kros et al., 1998; Marcotti et al., 2004).

\section{MET current}

Deflection of the hair bundle from its resting position alters the tension between the interciliary tip-links, which leads to a modulation of the number of open MET channels. Hence the deflection of the stereocilia bundle modulates the apical conductance of the IHC, generating a MET current. In vitro, following a step-displacement of the ciliary bundle, the MET current was shown to exhibit exponential adaptation (Howard and Hudspeth, 1987). A recent study by Corns et al. (2014) shows that at physiological levels of extracellular $\mathrm{Ca}^{2+}$, only very small (and relatively slow) MET current
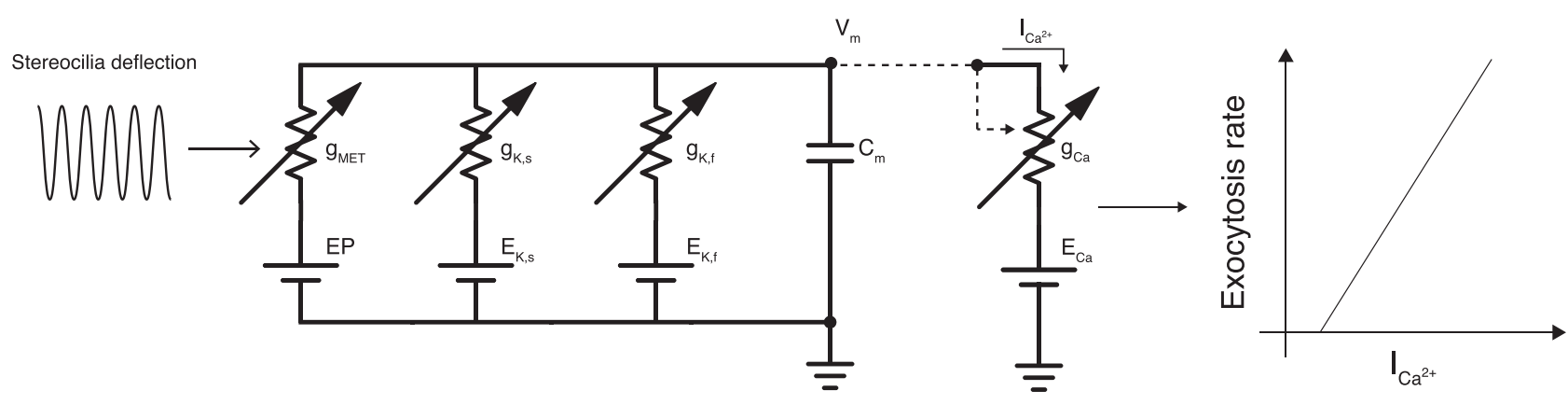

FIG. 1. Schematic of the model employed for this study. A model of the IHC that includes the voltage-dependent outward $\mathrm{K}^{+}$current is employed to compute the membrane potential in response to sinusoidal deflections of the ciliary bundle. The IHC potential controls the activation of the voltage-gated Ca ${ }^{2+}$ channels at the synapses. The $\mathrm{Ca}^{2+}$ current controls the rate of neurotransmitter release through a simplified model of exocytosis. 
adaptation is observed in mammalian IHC during fluid-jet stimulation of the ciliary bundle. For this reason, we neglect the effects of MET current adaptation in this analysis.

The activation of the MET conductance is well described by a three-state Boltzmann function of ciliary displacement (Kros et al., 1992); however, for small enough values of stereocilia deflection the activation can equally be expressed as a single Boltzmann nonlinearity. This simplification reduces the number of parameters of the transduction nonlinearity from 4 to 2 .

Adopting a first-order activation ${ }^{1}$ with a time constant $\tau_{\mathrm{MET}}=50 \mu$ s (Kennedy et al., 2003), the transducer conductance can be calculated from the ciliary deflection $(\mu)$ by treating the MET channels as standard ion channels,

$$
\begin{aligned}
& n_{\mathrm{MET}, \infty}=\frac{1}{1+e^{-\left(\mu-x_{0}\right) / s}}, \\
& n_{\mathrm{MET}}+\tau_{\mathrm{MET}} \frac{\mathrm{d} n_{\mathrm{MET}}}{\mathrm{d} t}=n_{\infty}, \\
& g_{\mathrm{MET}}(\mu)=n G_{\mathrm{MET}},
\end{aligned}
$$

where $G_{\mathrm{MET}}$ is the maximum transducer conductance, $n_{\mathrm{MET}, \infty}$ represents the steady-state activation of the MET channels given $\mu$, and $n_{\mathrm{MET}}$ the instantaneous fraction of open MET channels. Here we adopt $G_{\max }=30 \mathrm{nS}, s=16 \mathrm{~nm}$ and $x_{0}=35 \mathrm{~nm}$ based on the recordings by Jia et al. (2007) from basal IHCs of the mature gerbil in a hemicochlea.

Finally, since the reversal potential of the MET channels is close to $0 \mathrm{mV}$, the transduction current can be expressed as

$$
I_{\mathrm{MET}}=g_{\mathrm{MET}}(\mu)\left(V_{\mathrm{m}}-\mathrm{EP}\right),
$$

where EP is the endocochlear potential. Here, we use EP $=90 \mathrm{mV}$.

\section{Outward potassium currents}

In addition to the transducer current, the presence of outward $\mathrm{K}^{+}$currents has been shown to play a major role in the hair-cell physiology in vitro (Hudspeth and Lewis, 1988; Kros and Crawford, 1990; Marcotti et al., 2004; Johnson et al., 2011; Johnson, 2015). In mature high-frequency IHCs, the total voltage-gated $\mathrm{K}^{+}$current is largely determined by a fast $\left(I_{\mathrm{K}, \mathrm{f}}\right)$ and a slowly activating outward current $\left(I_{\mathrm{K}, \mathrm{s}}\right)$ (Marcotti et al., 2004; Johnson, 2015).

The total $\mathrm{K}^{+}$current can be described by

$$
I_{\mathrm{K}}=g_{\mathrm{K}, \mathrm{f}}\left(V_{\mathrm{m}}-E_{\mathrm{K}, \mathrm{f}}\right)+g_{\mathrm{K}, \mathrm{s}}\left(V_{\mathrm{m}}-E_{\mathrm{K}, \mathrm{s}}\right),
$$

where $g_{\mathrm{K}, \mathrm{f}}$ and $g_{\mathrm{K}, \mathrm{s}}$ are the voltage-dependent conductances of the fast and slow $\mathrm{K}^{+}$channels, respectively, and $E_{\mathrm{K}, \mathrm{f}}=-71 \mathrm{mV}$ and $E_{\mathrm{K}, \mathrm{s}}=-78 \mathrm{mV}$ are the reversal potentials of the fast and slow channels, respectively (Kros and Crawford, 1990).

The activation kinetics of both fast and slow $\mathrm{K}^{+}$channels are well described by two voltage-dependent time constants (Kros and Crawford, 1990), but a first-order equation has been shown to approximate the activation of the fast $\mathrm{K}^{+}$ channels well (Marcotti et al., 2004). This simplification has only a minimal impact on the results depicted here, as the second-order differential equation describing the kinetics of the $\mathrm{K}^{+}$current describes an over-damped oscillator [see Eq. (3) of Lopez-Poveda and Eustaquio-Martín, 2006], whose frequency response can be approximated with a first-order equation. Additionally, because the principal time constants of the activation of the $\mathrm{K}^{+}$channels show very small variations for membrane potentials around the resting value (see Fig. 12 of Kros and Crawford, 1990), we regard them as constant.

Based on these arguments, the activation kinetics of the two $\mathrm{K}^{+}$channels can be described by the following equations:

$$
\begin{aligned}
& n_{\mathrm{K}, \mathrm{f} / \mathrm{s}, \infty}=\frac{1}{1+e^{-\left(V_{\mathrm{m}}-V_{0.5}\right) / \mathrm{s}}}, \\
& n_{\mathrm{K}, \mathrm{f} / \mathrm{s}}+\tau_{\mathrm{K}, \mathrm{f} / \mathrm{s}} \frac{\mathrm{d} n_{\mathrm{K}, \mathrm{f} / \mathrm{s}}}{\mathrm{d} t}=n_{\mathrm{K}, \mathrm{f} / \mathrm{s}, \infty}, \\
& g_{\mathrm{K}, \mathrm{f} / \mathrm{s}}=n_{\mathrm{K}, \mathrm{f} / \mathrm{s}} G_{\mathrm{K}, \mathrm{f} / \mathrm{s}} .
\end{aligned}
$$

The half-activation potential $\left(V_{0.5}\right)$ depends on the intracellular level of $\mathrm{Ca}^{2+}$ buffers (Marcotti et al., 2004). Johnson et al. (2011) estimated the activation curve of the total $\mathrm{K}^{+}$ outward currents in mature gerbil IHCs at the physiologically equivalent level of $\mathrm{Ca}^{2+}$ buffers ( $1 \mathrm{~mm}$ EGTA). In order to employ these estimates from mature cells in a highly controlled preparation, we assume that the two channels share the same activation curve. This assumption is justified by the similar voltage-gating for the two channels (see the Boltzmann fits by Zeddies and Siegel, 2004 on the data in Kros and Crawford, 1990). We employ $G_{\mathrm{K}, \mathrm{f}}=G_{\mathrm{K}, \mathrm{s}}=230$ $\mathrm{nS}, V_{0.5}=-31 \mathrm{mV}$ and $s=10.5 \mathrm{mV}$, based on the study of Johnson et al. (2011).

At body temperature, the fast-activating $\mathrm{K}^{+}$channels activate with principal time constants $\left(\tau_{\mathrm{K}, \mathrm{f}}\right)$ of about $0.2 \mathrm{~ms}$ in the mature mouse (Kros et al., 1998) with a holding potential of $-25 \mathrm{mV}$. In the mature gerbil, the principal time constant is $0.5 \mathrm{~ms}$ measured at room temperature and with a holding potential of $-37 \mathrm{mV}$ (Marcotti et al., 2004). Using a Q10 factor of 1.76 (Johnson et al., 2011), this time constant becomes $0.25 \mathrm{~ms}$ at body temperature. These values are similar to those estimated in guinea pig cells at body temperature $^{2}$ at similar holding potentials (Kros and Crawford, 1990). We therefore utilize $\tau_{\mathrm{K}, \mathrm{f}}=0.3 \mathrm{~ms}$ based on Fig. 12 of Kros and Crawford (1990).

The slow-activating $\mathrm{K}^{+}$channels activate with a principal time constant of about $4 \mathrm{~ms}$ in mature mouse cells with a holding potential of $-25 \mathrm{mV}$ at body temperature (Kros et al., 1998). This value is similar to that estimated in the guinea pig utilizing the same holding potential (Kros and Crawford, 1990), which corresponds to a value of about $8 \mathrm{~ms}$ near the cell's resting potential. We therefore adopt $\tau_{\mathrm{K}, \mathrm{s}}=8 \mathrm{~ms}$.

\section{B. Electrical to neural transduction}

\section{Whole-cell $\mathrm{Ca}^{2+}$ current}

The rate of exocytosis at the IHC ribbon synapse is regulated by $\mathrm{Ca}^{2+}$ influx through L-type $\left(\mathrm{Ca}_{\mathrm{V}} 1.3\right)$ channels 
(Platzer et al., 2000; Brandt et al., 2003). The kinetics of the $\mathrm{Ca}_{\mathrm{V}} 1.3$ channels are effectively described by second-order activation and second-order inactivation (Johnson and Marcotti, 2008). The faster inactivation time constant is roughly $50 \mathrm{~ms}$, implying that the inactivation of the $\mathrm{Ca}_{\mathrm{V}} 1.3$ has a negligible effect on the experimental derivation of neural tuning curves in which the stimulus duration is $50 \mathrm{~ms}$.

The time course of activation is voltage dependent (Johnson and Marcotti, 2008); however, it may be considered constant for small excursions of the IHC receptor potential. The voltage-dependent conductance of the $\mathrm{Ca}^{2+}$ channels $\left(g_{\mathrm{Ca}}\right)$ can therefore be expressed as

$$
\begin{aligned}
& m_{\infty}=\left(1+e^{-\left(V_{\mathrm{m}}-V_{0.5}\right) / s}\right)^{-1 / 2}, \\
& m+\tau_{\mathrm{Ca}} \frac{\mathrm{d} m}{\mathrm{~d} t}=m_{\infty}, \\
& g_{\mathrm{Ca}}\left(V_{\mathrm{m}}\right)=G_{\mathrm{Ca}} m^{2} .
\end{aligned}
$$

We adopt $s=7.5 \mathrm{mV}, V_{0.5}=-25 \mathrm{mV}, G_{\mathrm{Ca}}=4.1 \mathrm{nS}$, and $\tau_{\mathrm{Ca}}=0.2 \mathrm{~ms}$ based on the recordings from basal IHCs of the mature gerbil (Johnson and Marcotti, 2008). It should be noted that the value of $G_{\mathrm{Ca}}$ is of no importance for the following analysis as it is a scaling constant that allows the predicted values of the whole-cell $\mathrm{Ca}^{2+}$ current to fall within the physiological range.

The total (whole-cell) current flowing through the $\mathrm{Ca}_{\mathrm{v}}$ 1.3 channels is then given by

$$
I_{\mathrm{Ca}}=g_{\mathrm{Ca}}\left(V_{\mathrm{m}}-E_{\mathrm{Ca}}\right),
$$

where $E_{\mathrm{Ca}}=45 \mathrm{mV}$ is the reversal potential of the $\mathrm{Ca}_{\mathrm{v}} 1.3$ channels.

\section{2. $\mathrm{Ca}^{2+}$ controlled exocytosis}

In the physiological range of the IHC membrane potential, the relationship between the whole-cell $\mathrm{Ca}^{2+}$ current $I_{\mathrm{Ca}}$ and the exocytosis rate in mammalian IHCs is approximately linear (Johnson et al., 2005; Goutman and Glowatzki, 2007), at least in high-frequency units (Johnson et al., 2008). Therefore, we express the relationship between the wholecell $\mathrm{Ca}^{2+}$ current $I_{\mathrm{Ca}}$ and the exocytosis rate at a single synapse $(k)$ through the following quasi-linear ${ }^{3}$ synaptic transfer function:

$$
k(t)=z \max \left(I_{\mathrm{Ca}}-I_{\mathrm{Ca}, \mathrm{th}}, 0\right),
$$

where $k(t)$ is expressed in release events per time unit, $I_{\mathrm{Ca}, \text { th }}$ is a fraction of the resting $\mathrm{Ca}^{2+}$ current representing the offset of the straight line relating $\mathrm{Ca}^{2+}$ influx and the exocytosis rate at individual synapses, and $z$ is a constant. The max function represents the fact that the exocytosis rate cannot be negative.

The two parameters in the synaptic transfer function account for the different dynamic ranges of exocytosis among different synapses. The offset in Eq. (14) predicts the persistence of a small $\mathrm{Ca}^{2+}$ current for membrane potentials more negative than those corresponding to the activation of the postsynaptic current, which was first reported in bullfrog
IHCs by Keen and Hudspeth (2006). The same phenomenon might be observed in mammalian hair cells, e.g., by comparing Figs. 3A1 and 3A2 of Goutman and Glowatzki (2007). Note that Eq. (14) can result from the first-order polynomial expansion of a nonlinear function relating $I_{\mathrm{Ca}}$ and $k$. Because the gating of the $\mathrm{Ca}^{2+}$ channels differs among different synapses (Frank et al., 2009; Ohn et al., 2016), the existence of a perfectly linear relationship between the whole-cell $\mathrm{Ca}^{2+}$ current and the exocytosis rate at a single synapse is very unlikely to exist.

Two conditions are needed to determine the parameters of Eq. (14) at individual synapses. The conditions employed here are: (i) $k$ matches the AF discharge rate at rest and (ii) the peak value of $k$ at saturation matches the peak AF discharge rate measured at very high sound pressure levels (SPLs).

The first condition is motivated by the fact that a single release event is sufficient to drive an action potential in the afferent AF when it is not in a refractory state (Siegel, 1992; Rutherford et al., 2012), and hence the rate of neurotransmitter release and $\mathrm{AF}$ discharge rate are closely related. Estimating the value of the exocytosis rate corresponding to the AF spontaneous discharge rate by accounting for refractory effects through statistical models would be possible (e.g., Vannucci and Teich, 1978). However, as the higher AF spontaneous discharge rates are in the order of 100 spikes/s while the refractory time constants are shorter than $1 \mathrm{~ms}$ (see, e.g., Peterson et al., 2014), we assume that the difference between the spontaneous rate of exocytosis and that of the AF discharge is negligible for the present analysis.

The second condition is motivated by the linear relationship between the macroscopic $\mathrm{Ca}^{2+}$ current and the exocytosis rate at the onset of the electrical stimulation of the IHC (Goutman and Glowatzki, 2007). In principle, the parameters of Eq. (14) can be determined from the measured AF discharge rate at the onset of the response to acoustic stimulation at very high SPL, where the IHC responses can be considered saturated and little effects of synaptic depression and refractoriness are observed. Unfortunately, this fitting procedure can result in the peak of exocytosis rate being underestimated because the rise time of the mechanical drive to the IHC in vivo is larger than that of a voltage step of the receptor potential in vitro: whereas the rise time of a voltage step of the receptor potential is limited only by the electrical time constants of the IHC and of the experimental equipment, the rise time of the stereocilia drive is limited by the ramp applied to the acoustical stimulus (typically $1 \mathrm{~ms}$ long for tones) and by the time constants of the different mechanical elements involved in the transformation of the ear-canal pressure into deflection of the IHC stereocilia. Therefore, during the time the mechanical stimulation to the stereocilia reaches its maximum, some vesicles can be released with a significant probability, thereby limiting the measurable peak of the AF discharge rate.

Additionally, to the authors' best knowledge, there is no existing systematic characterization of the peak responses of AFs to very loud tones. To partially account for these two issues, a generous range of possible peak values of the exocytosis rate is employed in the numerical simulations. 
In this study, representative models of low (0.01-1 spikes/s), medium (1-18 spikes/s), and high (18-100 spikes/s) spontaneous rate $\mathrm{AF}$ are employed. The strength of $\mathrm{AF}$ responses at the onset strongly correlates with the AF spontaneous rate, with a peak-to-steady ratio of AF responses to 25-50 ms tone bursts spanning from 1 (low spontaneous-rate units) to above 8 (high spontaneous-rate units) (Rhode and Smith, 1985; Taberner and Liberman, 2005). By assuming steady-state $^{4}$ firing rates between 180 and 300 spikes/s (Zagaeski et al., 1994) and peak-to-steady ratios between 1 and 3, 2 and 6, and 5 and 9, peak exocytosis rates of 180-900, 360-1500, and 900-2700 spikes/s are obtained for low, medium, and high spontaneous rate units, respectively.

\section{Determination of threshold levels}

The neural threshold criterion employed in this study is based on a widely adopted experimental procedure (Liberman, 1978). In short, a $50 \mathrm{~ms}$ acoustic tone is presented to the ear, and the activity of the auditory nerve is recorded during the tone presentation and the following $50 \mathrm{~ms}$. The neural threshold is defined as the minimum sound level that can consistently triggers one spike more during the tone presentation than during the following $50 \mathrm{~ms}$. By neglecting the role of synaptic depression in this procedure (see below), the neural threshold corresponds to an increase of 20 spikes/s above the considered AF spontaneous rate during the tone presentation. Similarly, the threshold criterion employed in this study corresponds to the minimum amplitude of ciliary deflection sufficient to drive the average exocytosis rate [computed using Eq. (14)] to 20 release events/s above its resting value. This assumption is justified on the basis that the present study focuses on levels of neural excitation corresponding to the neural threshold. For such levels of excitation only one extra spike over spontaneous activity can be registered during the threshold determination procedure. Because a single exocytotic event is sufficient to trigger an action potential in the afferent fiber (Siegel, 1992; Rutherford et al., 2012), only a single extra release of neurotransmitter is observable in the threshold determination procedure, implying that synaptic depression does not greatly affect the estimation of the neural thresholds.

\section{RESULTS}

\section{A. Simulations of the macroscopic $\mathrm{Ca}^{2+}$ current}

The macroscopic $\mathrm{Ca}^{2+}$ current can be simulated by imposing a sinusoidal deflection of the ciliary bundle in Eq. (5). Figure 2 shows the waveforms of the MET current, the IHC membrane potential and the $\mathrm{Ca}^{2+}$ current for a $50 \mathrm{~ms}$ sinusoidal deflection of the IHC stereocilia of $40 \mathrm{~nm}$ for different frequencies $(500 \mathrm{~Hz}, 2 \mathrm{kHz}, 8 \mathrm{kHz})$. The dashed horizontal lines indicate the average levels over the stimulus presentation.

The predicted membrane resting potential (middle row) for the selected parameters is $-59 \mathrm{mV}$. Determining the precise value of the IHC resting potential experimentally in vivo is practically impossible, as the electrode impalement creates an unknown leakage current that affects the activations of the various ion channels. Additionally, the value of the IHC resting potential depends on the magnitude of the MET current at rest, which in turns depends on the $\mathrm{Ca}^{2+}$ concentration in the proximity of the stereocilia and the presence of mobile $\mathrm{Ca}^{2+}$ buffers (Ricci et al., 1998; Johnson et al., 2011; Corns et al., 2014).

Zeddies and Siegel (2004) calculated a resting membrane potential of $-64 \mathrm{mV}$. However, they assumed smaller magnitudes for the MET conductances than those estimated
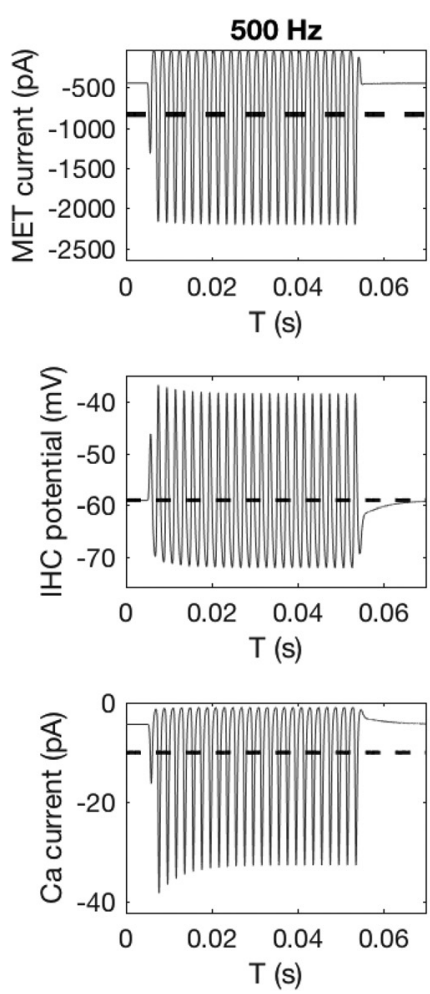

$2000 \mathrm{~Hz}$
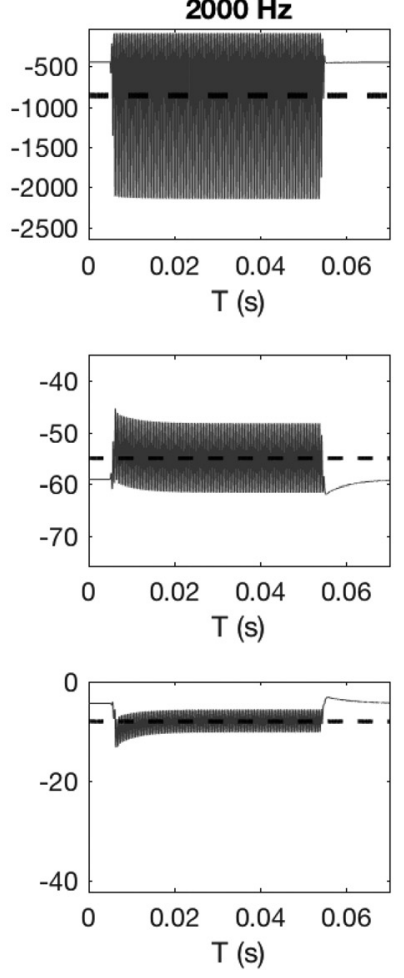

$8000 \mathrm{~Hz}$
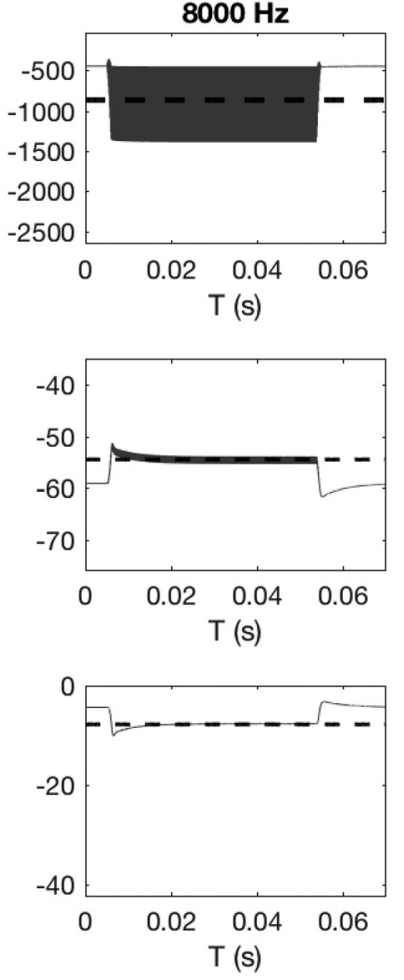

FIG. 2. Simulated IHC responses to $40 \mathrm{~nm}$ sinusoidal ciliary deflections of different frequencies. From top to bottom: the MET current, the membrane potential and the whole-cell $\mathrm{Ca}^{2+}$ current. The dashed lines indicate the average value (DC component) of the curves during the stimulation of the hair bundle. 
more recently for mature IHCs at physiological levels of extracellular $\mathrm{Ca}^{2+}$ concentration (Jia et al., 2007; Johnson et al., 2011). The predicted resting potential of the present model is in agreement with the findings by Johnson et al. (2011), who estimated it to be between -60 and $-55 \mathrm{mV}$ in mature gerbils at physiological levels of extracellular $\mathrm{Ca}^{2+}$.

The activation curve of the MET channels [Eq. (5)] distorts the sinusoidal ciliary deflection, so the MET current can be thought of as a sum of sinusoids (AC components) and a continuous (DC) component. The non-instantaneous activation of the MET channels attenuates the higher harmonics of the AC component of the MET current, similarly to a low-pass filter with a cut-off frequency of $3.2 \mathrm{kHz} .^{5}$ The action of the IHC membrane [Eq. (1)] is similar to that of a low-pass filter, which attenuates the high-frequency components of the receptor potential. The time constant of the IHC-associated low-pass filter depends on the activation of the $\mathrm{K}^{+}$current, and its value at rest is $0.4 \mathrm{~ms}$. The action of the outward $\mathrm{K}^{+}$currents attenuates the DC component and further distorts the waveform of the receptor potential for the lowest frequency stimuli. Small sags are visible at the onset and the offset of the stimuli, which have been previously predicted by modeling studies (Zeddies and Siegel, 2004; Lopez-Poveda and Eustaquio-Martín, 2006).

The $\mathrm{Ca}^{2+}$ current $\left(I_{\mathrm{Ca}}\right)$ in the bottom row of Fig. 2 is a distorted low-pass-filtered version of the receptor potential. This distortion is caused by the activation of the $\mathrm{Ca}^{2+}$ channels, which converts part of the AC component of the receptor potential into a DC component of $I_{\mathrm{Ca}}$. The second-order activation kinetics of $I_{\mathrm{Ca}}$ [Eq. (10)] is analogous to a secondorder low-pass filter with a cut-off frequency of $800 \mathrm{~Hz}$, which attenuates the high-frequency components of $I_{\mathrm{Ca}}$.

The lower panels in Fig. 2 show that for the same level of mechanical excitation of the IHC stereocilia, the average component of $I_{\mathrm{Ca}}$ differs slightly depending on the frequency of the ciliary oscillation. To further investigate the relationship between the frequency of the bundle oscillation and the level of $\mathrm{Ca}^{2+}$ influx at the synapse, Fig. 3(A) shows the average value of $I_{\mathrm{Ca}}$ as a function of the frequency with which the stereocilia vibrate (solid line). The figure shows that the average level of $\mathrm{Ca}^{2+}$ influx at the synapse is fairly independent of the frequency of the bundle's oscillation, whose level lies within $\pm 1.5 \mathrm{~dB}$ around its median value for all frequencies. The same figure shows the peak-to-peak value of $I_{\mathrm{Ca}}$ (dashed-line), which is a measure of the level of the AC component of $\mathrm{Ca}^{2+}$ influx. The AC component of the $\mathrm{Ca}^{2+}$ influx at the synapse is approximately constant at low frequencies, exhibiting a weak resonance around $500 \mathrm{~Hz}$ caused by the interaction of the IHC membrane time constant and the activation of the fast outward $\mathrm{K}^{+}$current. The AC component of $I_{\mathrm{Ca}}$ rapidly decays for frequencies above 1 $\mathrm{kHz}$. This is not surprising as the mechanical-to-neural transduction process involves several cascades of nonlinearities followed by low-pass filtering. As the exocytosis rate at the IHC ribbon synapse is approximately proportional to $I_{\mathrm{Ca}}$, the phase-locking properties of the afferent fibers greatly depend on the magnitude of the AC component of $I_{\mathrm{Ca}}$. To further test the accuracy of the model prediction of the phaselocking limits of the AF responses, Fig. 3(B) compares the range of the predicted vector strengths of the AF responses against those measured in the base of the chinchilla by Temchin and Ruggero (2010). Although our model does not account for synaptic depression or refractoriness (that might affect the vector strength of AF responses), the model is in good agreement with the reference data.

\section{B. Estimating the relationship between neural and stereocilia frequency tuning}

Due to the heterogeneity of $\mathrm{Ca}^{2+}$ signaling at the synapse (Frank et al., 2009; Wong et al., 2013; Ohn et al., 2016) and AF discharge rates (Liberman, 1978), obtaining a
(A)

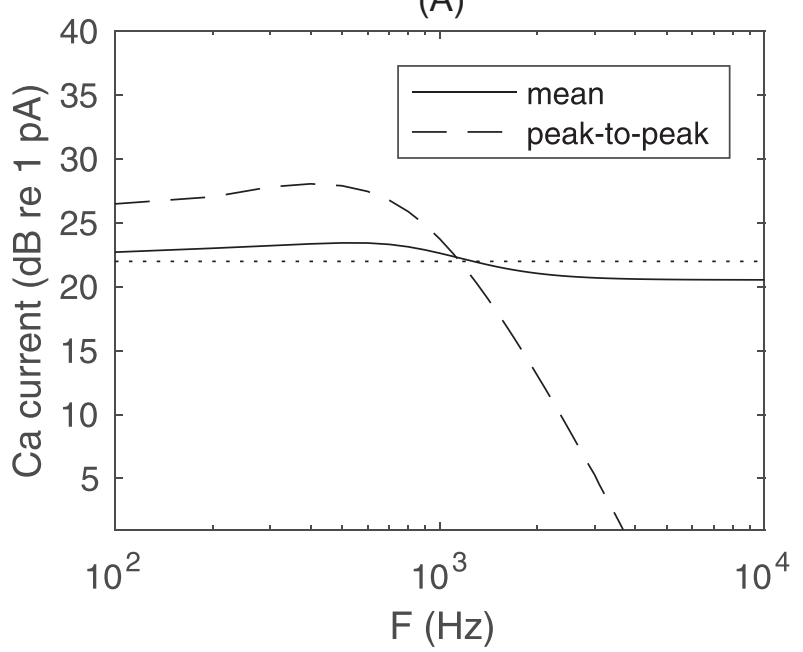

(B)

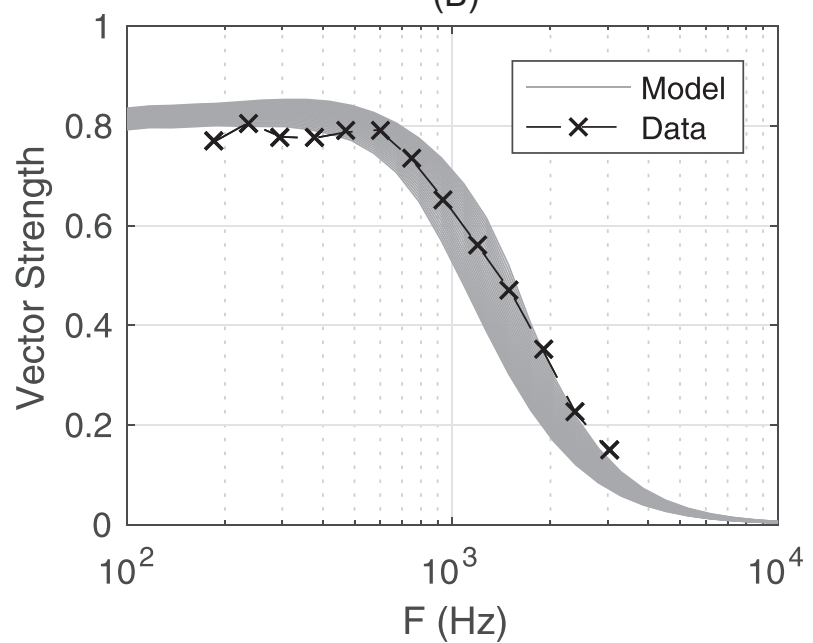

FIG. 3. (A) Simulated average (solid line) and peak-to-peak values (dashed line) of the $\mathrm{Ca}^{2+}$ current at the IHC synapse as a function of the frequency of the stereocilia oscillation (oscillation amplitude of $40 \mathrm{~nm}$ ). The dotted line indicates the median value of the average Ca ${ }^{2+} \mathrm{current}(\mathrm{B}) \mathrm{Predicted}$ range of the vector strength of the AF responses as a function of the frequency of the stereocilia oscillation (in grey) vs the average vector strength of AF responses in the chinchilla base [data from Fig. 7(A) of Temchin and Ruggero, 2010]. In the model, the vector strength of the AF responses depends upon the rectification strength at the synapse, which is controlled by the parameter $I_{\mathrm{Ca}, \text { th }}$ in Eq. (14). Therefore the range of predicted vector strengths was obtained by sweeping $I_{\mathrm{Ca} \text {,th }}$ from its minimum to its maximum value in 30 steps. 
precise function relating the exocytosis rate and $\mathrm{Ca}^{2+}$ current at the individual synapse it is currently not possible. Consequently, we tested the model predictions using a wide range of parameters to describe the synaptic transfer function Eq. (14). Figure 4(A) plots the estimated amplitude of the stereocilia deflection necessary to drive the different AF models above the threshold as a function of the stimulus frequency. Depending on the combination of the parameters of the synaptic transfer function, there is a substantial heterogeneity of the $\mathrm{AF}$ thresholds. To test whether the model can account for the heterogeneity of AF thresholds reported in experimental studies, the amplitude of the stereocilia deflection corresponding to neural threshold is computed for 200 AF models with randomly distributed parameters (spontaneous rate and peak of exocytosis rate) with a driving frequency of $8 \mathrm{kHz}$. These results are plotted as a function of spontaneous rate using a logarithmic scale in Fig. 4(B) along with the data of Fig. 4(B) of Winter et al. (1990). The predicted amplitudes of the stereocilia deflection necessary to drive the most and least sensitive fibers differ by $12 \mathrm{~dB}$. This difference in combination with a compressive growth of the mechanical responses with the SPL of $0.2-0.3 \mathrm{~dB} / \mathrm{dB}$ at $\mathrm{CF}$ (Robles and Ruggero, 2001; Ren et al., 2016b) predicts a range of AF thresholds between 40 and $60 \mathrm{~dB}$ SPL, which is in good agreement with experimental data (Liberman, 1978; Winter et al., 1990). Additionally, the figure shows that the range of variability of the parameters adopted is adequate to mimic the heterogeneity of AF thresholds and their dependence on the unit's spontaneous rate.

The data in Fig. 4(A) show that for all the simulated cases, a smaller deflection of the ciliary bundle is necessary to drive the AF above threshold at low frequencies than at higher frequencies. To better visualize this difference, the curves in Fig. 4(A) are normalized, and their reciprocals are plotted in Fig. 4(C). The resulting curves provide a measure of the frequency sensitivity of the transduction process at individual synapses and reflect the difference between the frequency tuning of the stereocilia and that of the AF.

Depending on the exact combination of the model parameters, there is substantial variability in the frequency sensitivity of the AF. Despite this variability, the curves retain the same shape, i.e., they are well described by the same piecewise function [dashed lines in Fig. 4(C)]. This function, which will be referred to as the synaptic frequency sensitivity $S(f)$, shows a plateau below a corner frequency $f_{\mathrm{c}}$ and exponentially decays for frequencies above $f_{\mathrm{c}}$,

$$
S(f)= \begin{cases}P, & f<f_{\mathrm{c}}, \\ P e^{\left(f_{\mathrm{c}}-f\right) / f_{\mathrm{c}}}, & f \geq f_{\mathrm{c}},\end{cases}
$$

where $P$ indicates the peak sensitivity at low frequencies, corresponding to the level of the low-frequency plateau. For all cases shown in Fig. 4(C), a fixed $f_{\mathrm{c}}$ of $450 \mathrm{~Hz}$ captures the simulated data with $1 \mathrm{~dB}$ accuracy. The largest deviation is shown for the unit with the highest threshold level for which the best-fitting value of $f_{\mathrm{c}}$ is larger $\left(f_{\mathrm{c}}=500 \mathrm{~Hz}\right.$, data not shown). This deviation is a consequence of the nonlinear activation of the IHC membrane currents, which yields a stimulus-level-dependent variation in the effective time constant of the IHC. Since the deviation is small, it is neglected from the following analysis.

Although the data in Fig. 4(C) show that the smallest values of $P$ are associated with high spontaneous-rate units, no simple relationship can be drawn between the spontaneous rate and $P$. To investigate the range of variability of $P$ and its dependence on the characteristics of individual AFs, we computed it for 200 AF models with randomly distributed parameters in $0.2 \mathrm{~dB}$ steps.

Figure 5(A) plots the peak value of the frequencysensitivity function $[P$ in Eq. (15)] against the spontaneous rate of the simulated AFs. The values of $P$ vary from 1.4 to $9.2 \mathrm{~dB}$, with a median value of $6.2 \mathrm{~dB}$ and an interquartile range of $1.2 \mathrm{~dB}$. By categorizing the AFs according to their spontaneous rate, small differences in $P$ can be noted between low and medium spontaneous-rate units, where $P$ spans from 3 to $9.2 \mathrm{~dB}$. High spontaneous-rate units, on the other hand, exhibit the lowest values of $P(1.4-9.2 \mathrm{~dB})$, and $P$ decreases with an increasing spontaneous rate.

Figure 5(B) plots the values of $P$ against the maximum peak discharge rate associated with the different AF models. This figure shows that $P$ increases with the peak exocytosis
(A)

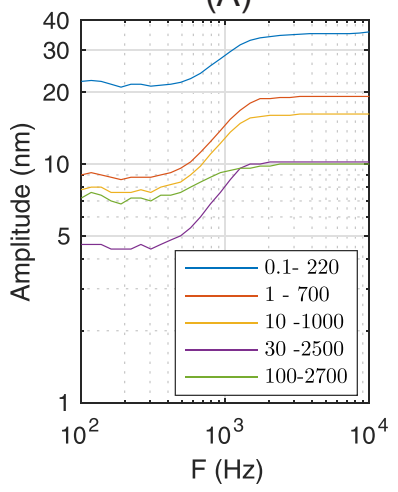

(B)

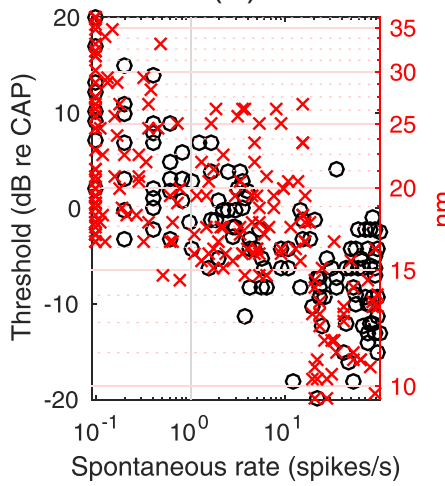

(C)

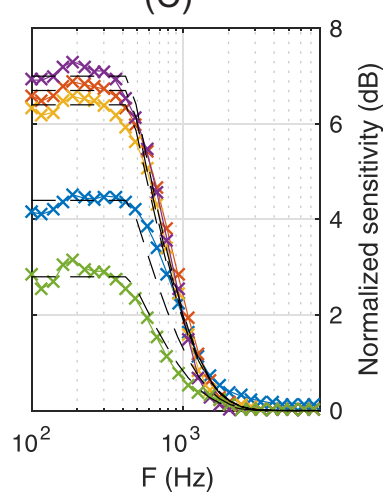

FIG. 4. (Color online) (A) Amplitude of ciliary displacement corresponding to the threshold level of the afferent AF. The curves indicate the stereocilia amplitude necessary to drive the average discharge rate of the afferent AF to 20 spikes/s above spontaneous rate. The different lines represent different AF models, whose spontaneous and peak-rate are indicated in the legend. (B) Model-based prediction of stereocilia deflection corresponding to the neural threshold (red markers $x$ ) plotted on top of the neural threshold level of the guinea-pig AFs in Fig. 4(B) of Winter et al. (1990) (black markers $\bigcirc$ ), as a function of AF spontaneous rate. The simulated data consist of 200 AF models whose parameters were randomly distributed (see Sec. II B 2). (C) Synaptic frequency-sensitivity for different AF models. These curves are obtained by normalizing and reciprocating the data in (A). The connected symbols indicate the model predictions, while the dashed lines indicate the fitting curves obtain through Eq. (15). 
(A)

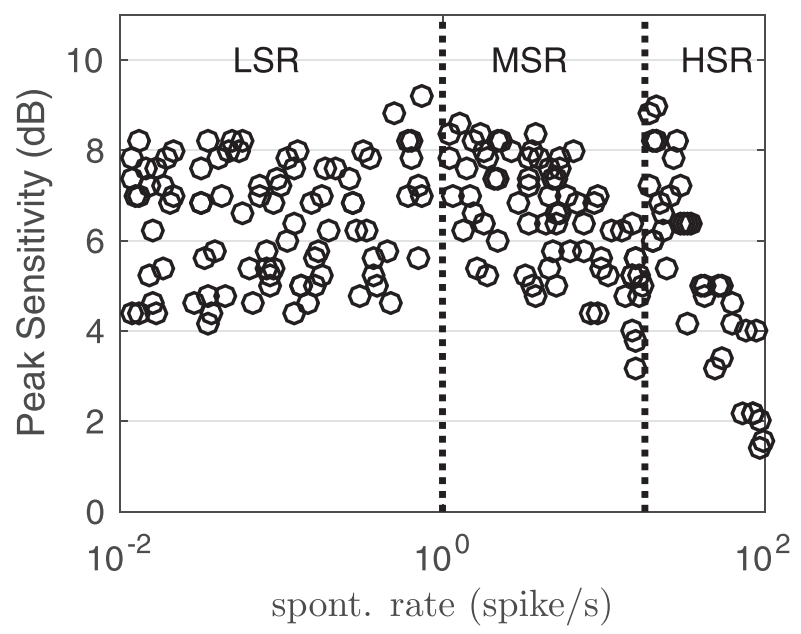

(C)

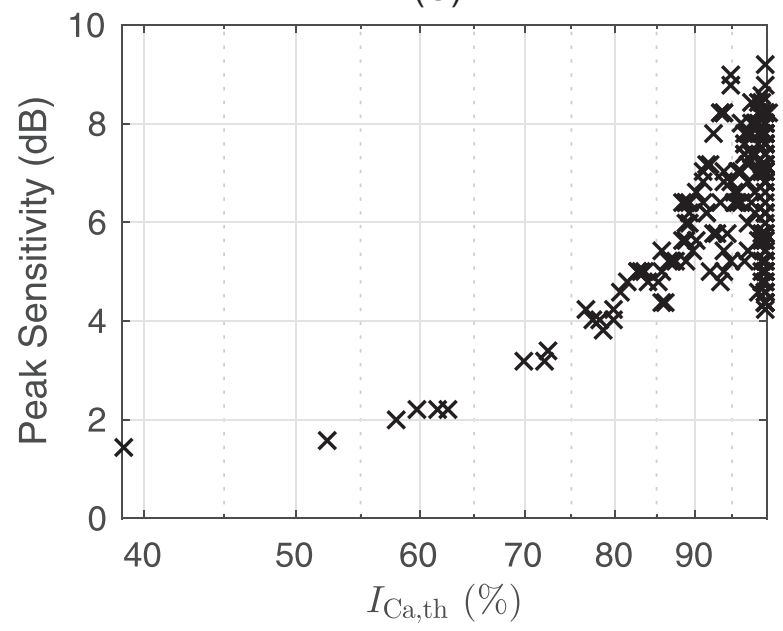

(B)

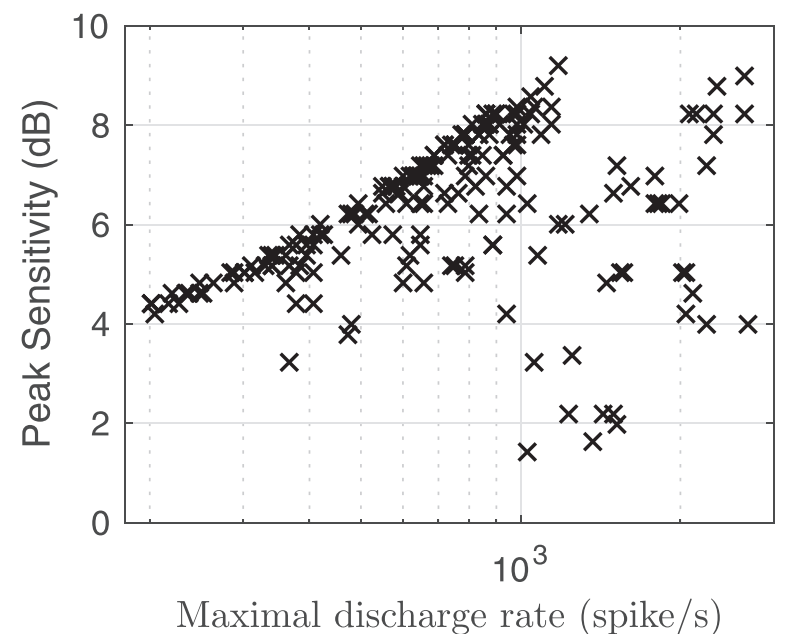

(D)

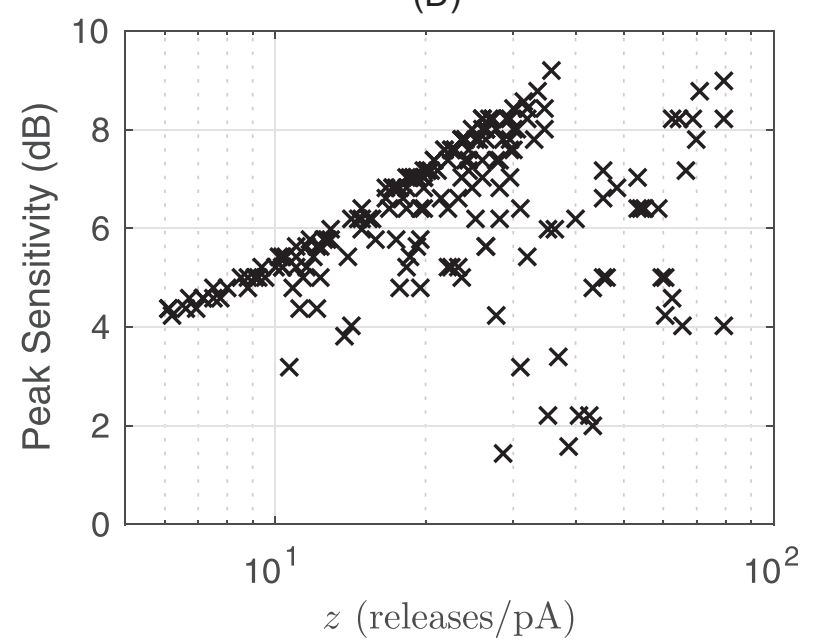

FIG. 5. Estimation of the peak of the frequency-sensitivity function. The frequency sensitivity at individual synapses is found to be well approximated by a low-frequency plateau [Eq. (15)] exponentially decaying above $450 \mathrm{~Hz}$. The peak $P$ of the synaptic frequency-sensitivity (corresponding to the level of the low-frequency plateau) is computed for $200 \mathrm{AF}$ models, whose parameters are randomly distributed. The simulated values of $P$ are plotted against (A) the spontaneous and (B) maximal rate of exocytosis; (C) the offset and (D) slope of the synaptic transfer function Eq. (14).

rate up to about 800 spikes/s. Above this value, there is no apparent correlation between $P$ and the peak exocytosis rate.

\section{Estimation of the tuning of stereocilia in vivo}

The frequency tuning of the IHC stereocilia can be estimated from the frequency tuning of the AF by accounting for the frequency sensitivity of the transduction process. For units in the basal region of the mammalian cochlea, the stereocilia tuning can be estimated by adding the synaptic frequencysensitivity function of Eq. (15) to the neural tuning curves. ${ }^{6}$

This method is applied to the recordings from the chinchilla base by Narayan et al. (1998). These recordings were selected because they provide measurements from the AF and the $\mathrm{BM}$ in the same cochlea. Figure 6 shows the reference data [Fig. 1(A) of Narayan et al., 1998] together with three estimates of the possible constant-stereocilia-deflection contours (dashed-lines) that are obtained by summing the synaptic frequency-sensitivity function to the neural tuning curves. Different low-frequency plateau levels $[P$ in Eq. (15)] for the synaptic frequency-sensitivity function were employed $(3,6$, and $9 \mathrm{~dB}$ ), covering the estimated range of variability of the considered AF, given its spontaneous rate of 11.4 spikes/s.

Above $2 \mathrm{kHz}$, the mechanical tuning of the stereocilia overlaps with the neural tuning curve, which closely resembles constant-BM-velocity contours. For frequencies between $500 \mathrm{~Hz}$ and $2 \mathrm{kHz}$, the difference between the tuning of the stereocilia and that of the BM velocity is smaller than the difference between the tuning of the BM velocity and that of the AF. According to the model, stereocilia deflection and BM velocity retain a very similar frequency tuning above $500 \mathrm{~Hz}$. For frequencies below $500 \mathrm{~Hz}$, the constant-stereocilia-deflection contours lie significantly below constant-BM-velocity contours, implying that the difference between the tuning of the BM velocity and that of the AF for these data cannot be accounted for by the transduction process alone.

\section{Heterogeneity of the frequency sensitivity of the synapses}

To investigate which model parameters are responsible for the variability in the peak value $P$ of the synaptic 


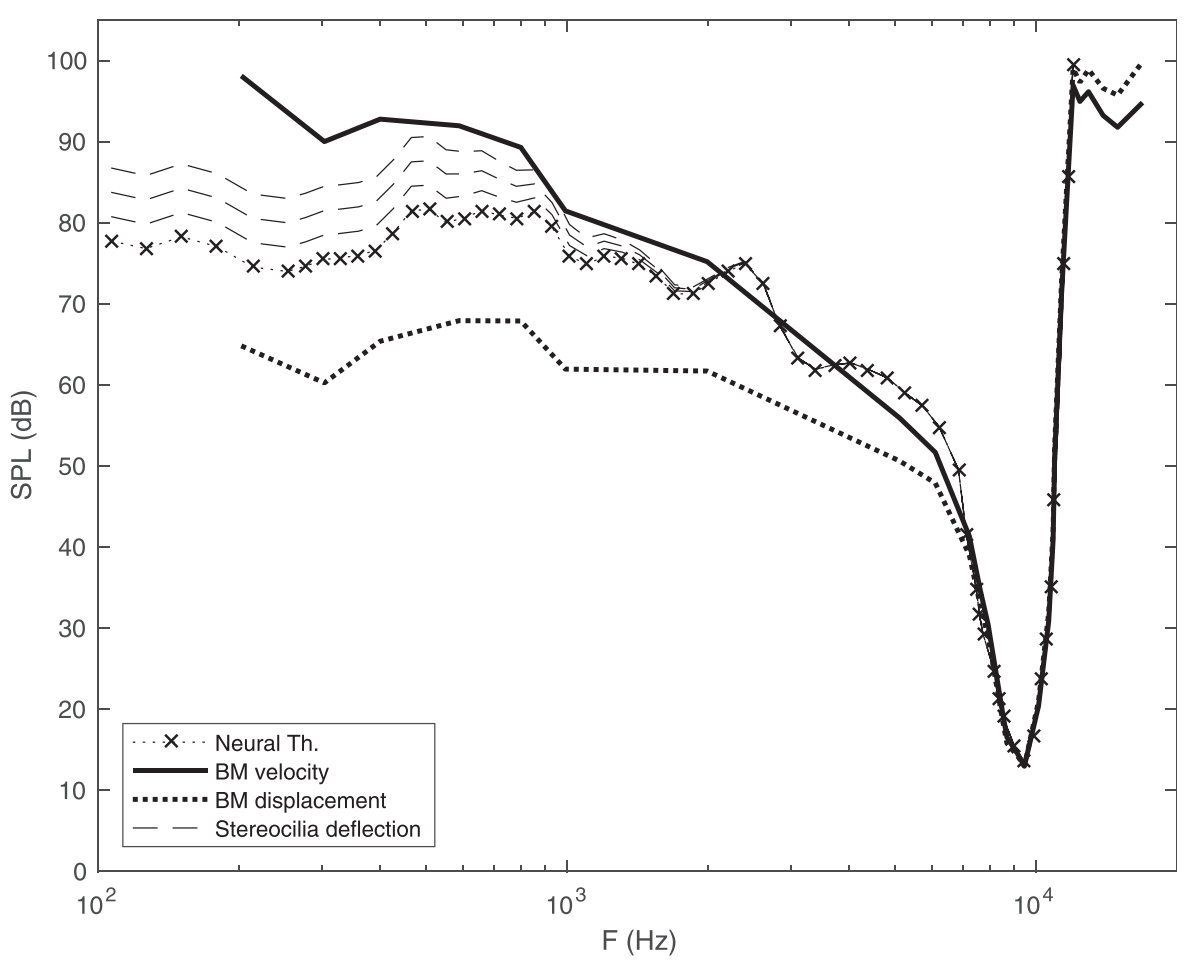

FIG. 6. Estimated stereocilia tuning from the neural tuning curves in Fig. 1(A) of Narayan et al. (1998). This figure shows the neural tuning curves and BM constant-velocity and constantdisplacement contours of the original study along with possible shapes of constant-stereocilia-deflection contours (dashed lines) obtained by accounting for the frequency sensitivity of the transduction process. The results of the present study show that the relationship between the frequency tuning of the auditory fibers and that of the stereocilia of the inner hair cell can be approximated with piecewise functions [Eq. (15)] with substantial variabilities in their low frequency peak value $P$ across different synapses. The different constant-stereocilia-deflection contours represent estimates using different values of $P(3,6$, and $9 \mathrm{~dB})$.

frequency sensitivity, Figs. 5(C) and 5(D) plot $P$ against the parameters of the synaptic transfer function Eq. (14), which were randomized in the numerical simulations.

The value of $P$ strongly depends on the parameters of the synaptic transfer function [Eq. (14)]. Figure 5(C) plots $P$ against the offset of the synaptic transfer function $\left(I_{\mathrm{Ca}, \mathrm{th}}\right)$, which is expressed as a fraction of the resting value of $I_{\mathrm{Ca}}$. This figure clearly indicates that the smallest $P$ values $(\leq 2 \mathrm{~dB})$ are associated with $I_{\mathrm{Ca} \text {,th }}$ below about $80 \%$ of the resting value of $I_{\mathrm{Ca}}$. In this range, $P$ and $I_{\mathrm{Ca} \text {,th }}$ are strongly correlated, whereas for $I_{\mathrm{Ca}, \mathrm{th}}$ above $80 \%$ the variability of $P$ increases abruptly.

Figure 5(D) plots $P$ against the slope of the synaptic transfer function $z$. The data distribution resembles that in Fig. 5(B), which is not surprising as $z$ and the maximal discharge rate are closely related parameters in the model [see Eq. (14)]. For the smallest values of $z, P$ shows little variability and lies around $4 \mathrm{~dB}$. With an increasing $z$, the data points spread vertically and demonstrate a poor correlation between $P$ and $z$.

The interplay of $I_{\mathrm{Ca} \text {,th }}$ and $z$ determines the frequency sensitivity at the synapse. For small values of $I_{\mathrm{Ca}, \mathrm{th}}$, there is only a small amount of rectification at the synapse, resulting in an average exocytosis rate that reflects the broad tuning of the DC component of the $\mathrm{Ca}^{2+}$ current. With increasing $I_{\mathrm{Ca} \text {, th }}$, the rectification of the synaptic transfer function becomes significant, and hence larger fractions of the AC component of $I_{\mathrm{Ca}}$ contribute to determine the average exocytosis rate. For AF models with high values of $I_{\mathrm{Ca}, \text { th }}, P$ depends on the threshold level, which in turn is determined by the value of $z$. For increasing threshold levels (equivalent to a decrease of $z$ ), the offset in the synaptic transfer function becomes increasingly negligible as it is much smaller than the DC level of $I_{\mathrm{Ca}}$ near threshold.

\section{E. Robustness of the model predictions}

To assess the dependence of the estimated synaptic frequency sensitivity on the parameters considered fixed up to this point, simulations of the mechanical-to-neural transduction were conducted with different values of the offset of the nonlinearity describing the activation of the MET channels [ $x_{0}$ in Eq. (2)]. This is a simple and effective way to estimate the robustness of our results to changes in the model parameters, because it simultaneously alters the membrane resting potential, the activation of the MET channels, the IHC time constant, and the activation of the $\mathrm{K}^{+}$and $\mathrm{Ca}^{2+}$ channels relative to the receptor potential.

Figure 7(A) plots the synaptic frequency sensitivity for three AF models (spontaneous rates 0.01,10, 60; peak rates 220, 1000, and 2700 spikes/s), simulated with $x_{0}=51 \mathrm{~nm}$ and $x_{0}=25 \mathrm{~nm}$ corresponding to $5 \%$ and $18 \%$ open MET channels at rest, and resting potentials of -63 and $-54 \mathrm{mV}$. In both cases, the shape of the frequency-sensitivity function is similar, with a higher corner frequency for the higher fraction of MET channels open at rest. This difference arises due to the different IHC time constants in the two cases. The low-frequency plateaus of the frequency sensitivity function are larger when more MET channels are open at rest. The range of variability of $P$ was estimated to be $0.8-5 \mathrm{~dB}$ and $3.4-11.8 \mathrm{~dB}$ in the two cases, on the basis of 200 simulated AF models.

To investigate the causes of this difference, we further computed the range of the variability of $P$ by manipulating the endocochlear potential [EP in Eq. (5)]. In this way, it was possible to alter the resting potential of the IHC, and consequently the relationship between activation of the $\mathrm{K}^{+}$and $\mathrm{Ca}^{2+}$ channels and the receptor potential without affecting the nonlinearity describing the activation of the MET channels. We impose $\mathrm{EP}=0$ and $\mathrm{EP}=200 \mathrm{mV}$, corresponding to IHC resting potentials of -63 and $-54 \mathrm{mV}$ as for the previous simulations. The manipulation of the EP yield $P$ in similar ranges for the two cases $(\mathrm{EP}=0 \mathrm{mV}, 1.8 \leq P \leq 8.8 \mathrm{~dB}$; $\mathrm{EP}=200 \mathrm{mV}, 2.4 \leq P \leq 9.2 \mathrm{~dB}$ ). Figure 7(B) compares the 

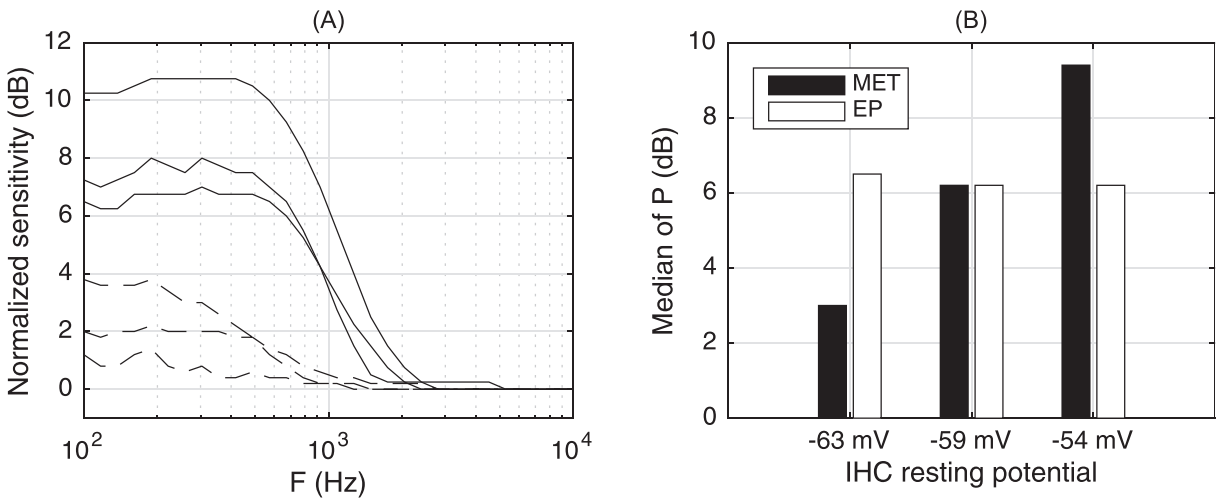

FIG. 7. Model robustness to the variation of the parameters. (A) The estimated synaptic frequency sensitivity is plotted for three AF models (different peak and spontaneous rate) when the offset of the Boltzmann nonlinearity describing the activation of the MET channel is modified $\left[x_{0}\right.$ in Eq. (2)]. $x_{0}=51 \mathrm{~nm}$ (dashed lines) yielding a resting potential of $-63 \mathrm{mV}$ and $x_{0}=25 \mathrm{~nm}$ (solid lines) corresponding to a receptor potential of $-54 \mathrm{mV}$. (B) Medians of the peak of the synaptic frequency sensitivity function $P$ of $200 \mathrm{AF}$ models when either the activation of the MET channel or the endocochlear potential (EP) were altered to obtain different values of the IHC resting potential. This figure shows that the predicted values of $P$ depend on the activation of the MET channels, while they are hardly affected by a $\pm 4.5 \mathrm{mV}$ perturbation of the IHC receptor potential per se, meaning that the model's predictions are fairly independent of the precise activation of the membrane currents relative to the receptor potential.

estimated median value of $P$ for the different values of the IHC resting potential, which were imposed either by altering the activation curve of the MET nonlinearity or the endocochlear potential. In the figure, the $-59 \mathrm{mV}$ case represents the median of the result drawn with the default model's parameter. Figure 7(B) shows that the median of the simulations remains approximately constant when the endocochlear potential is altered, whereas it increases with a more positive IHC resting potential when the activation curve of the MET channel is activated.

This result indicates that the estimated frequency sensitivity of the transduction process is fairly independent of small perturbations of the activation of the $\mathrm{K}^{+}$and $\mathrm{Ca}^{2+}$ channels relative to the receptor potential. On the other hand, the $P$ range depends heavily on the activation of the MET channels, because the AC/DC ratio of the IHC receptor potential increases with an increasing symmetry of the MET nonlinearity. This causes larger sensitivity differences between low and high frequencies for smaller values of the offset in Eq. (2).

\section{DISCUSSION}

\section{A. What drives the IHC stereocilia?}

The estimated frequency sensitivity of the transduction process was employed to derive constant-stereocilia-deflection contours from previously published neural tuning curves from the chinchilla base (Narayan et al., 1998). Even though the generalization of the results presented must be handled carefully because the proposed method is applied to a single data-set, we presented an example of applicability of the method to existing data.

The proposed method shows that the transduction process introduces a difference in the tuning of the stereocilia and that of the AF that is similar to the difference between the tuning of BM velocity and that of the AF above $500 \mathrm{~Hz}$ in the reference data. Below this frequency, constant-stereocilia-deflection contours lie well below constant-BM-velocity contours. These results indicate that, in the reference data, the frequency tuning of the mechanical drive to the IHC is similar to that of the BM velocity at high frequencies, whereas it shifts towards constant-BM-displacement contours at low frequencies. It is hard to reconcile this result with the classical view of stereocilia excited by BM velocity at low frequencies and BM displacement at high frequencies (e.g., Shamma et al., 1986; Cheatham and Dallos, 1999).

The results presented here indicate that at high frequencies the radial shear between TM and RL probably does not play a major role in deflecting the IHC stereocilia, as its magnitude is proportional to the relative displacement between TM and RL (Freeman and Weiss, 1990). Regardless of the precise nature of the mechanism driving the IHC stereocilia, our results strongly support the notion that the IHC stereocilia are free to vibrate without substantial attenuation up to their characteristic frequency (Zetes and Steele, 1997). The authors' interpretation of these results is that the fluid flow caused by a compression of the gap between the RL and the TM (see Guinan, 2012) controls the amount of stereocilia deflection at high frequencies, because the RL is similarly tuned to the BM (Chen et al., 2011; Ren et al., 2016a; Ren et al., 2016b) and a compression of the RL-TM gap proportional to the RL displacement would produce a fluid flow proportional to the RL velocity.

Several factors can be the source of the discrepancy between the tuning of the BM and that of the stereocilia at low frequencies. It is well-documented that the vibrations of the different elements of the $\mathrm{OC}$ are not trivially related to the vibrations of the BM (Mountain and Cody, 1999; Nowotny and Gummer, 2006; Karavitaki and Mountain, 2007; Guinan, 2012). Therefore, different mechanisms might be responsible in deflecting the IHC stereocilia at high and low frequencies.

\section{B. Limitations of the study}

The biggest limitation of this study is the impossibility of precisely establishing the parameters of the synaptic transfer function in vivo. The definition of a $\mathrm{Ca}^{2+}$-driven rate of exocytosis enabled us to establish a relationship between the 
mechanical excitation of the stereocilia and the AF firing rate for near-threshold level of excitation, where the effects of synaptic depression and refractoriness can be considered negligible. Whereas the $\mathrm{Ca}^{2+}$-driven exocytosis rate can be estimated when the IHC potential is at its resting value, establishing the range of values that it can assume during sustained stimulation is difficult. A representative range was estimated from the onset of AF responses to tonal stimulation at very high SPLs. In principle, at these levels, the IHC receptor potential can be considered saturated. Furthermore, the effects of synaptic depression or refractoriness should be small at the onset of the neural responses. The noninstantaneous rise-time of the mechanical excitation and the lack of a systematic characterization of AF discharge peak rates at very high SPLs might have led to underestimating the highest possible values of the driven exocytosis rate in vivo.

We conclude that a large error was not committed in estimating the range of variability of the $\mathrm{Ca}^{2+}$-driven exocytosis rate based on some properties of the model. First, the lowest predicted threshold levels correspond to a ciliary deflection of about $4 \mathrm{~nm}$. This value closely matches the smallest ciliary deflection that a hair cell can resolve (van Netten et al., 2003), leading to conclude that the slope of the synaptic transfer function was not significantly underestimated by the fitting procedure. Second, the estimated values of the peak exocytosis rate lead to the reproduction of the range of threshold levels of the AFs and their distribution among high, medium and low spontaneous activity units reported in the guinea pig by Winter et al. (1990).

The relatively large variability in the frequency sensitivity among different AFs, prevented a very accurate determination of the tuning of the stereocilia from published neural tuning curves. However, experiments can be designed to obtain a more precise estimate of the tuning of the stereocilia by determining possible parameters of the model for the measured AF. For example, this can be performed by determining the peak discharge rate in response to very loud clicks (over $120 \mathrm{~dB}$ ) for the considered AF, and then fitting the parameters of the synaptic transfer function to the measured data. These data would allow for the estimation of the saturation level of the $\mathrm{Ca}^{2+}$-driven exocytosis rate in vivo, limiting the effect of synaptic depression and refractoriness on the estimation due to the steeper onset of mechanical responses to clicks than to pure tones.

Another limitation of the study is the omission of MET current adaptation from the model equations. The fast component of MET current adaptation can in fact create a highpass filter effect on the MET current, as shown in the mature gerbil (Jia et al., 2007). At physiological levels of $\mathrm{Ca}^{2+}$, the fast time constant of MET current adaptation is larger than $1 \mathrm{~ms}$, and the amount of adaptation associated with such a fast component is small (Jia et al., 2007; Corns et al., 2014). By (generously) assuming that (i) the decrease of the MET current associated with the fast adaptation component is $40 \%$, (ii) the time constant is of $1 \mathrm{~ms}$, and (iii) by further describing the fast MET adaptation component through a first-order high-pass filter, we found that its inclusion in the model creates a maximal attenuation of the MET current of about $4 \mathrm{~dB}$ at very low-frequencies. At $150 \mathrm{~Hz}$, the attenuation produced is of about $1 \mathrm{~dB}$. Therefore, the exclusion of the MET current adaptation in the model equations introduces inaccuracies larger than $1 \mathrm{~dB}$ only for frequencies below $150 \mathrm{~Hz}$.

Last, the prediction of the model based on parameters stemming from in vitro data are hard to confirm experimentally in vivo. Nonetheless, the results presented here are supported by the in vivo recordings showing a predominant lowpass characteristic of the IHC and AF responses (e.g., Palmer and Russell, 1986). A partial confirmation of our predictions might be possible in vitro, e.g., by driving the stereocilia with a fluid-jet while counting the evoked post-synaptic potentials (EPSCs) in the afferent AF terminals.

\section{Heterogeneity of the frequency sensitivity of individual synapses}

The model-based estimation of the frequency sensitivity of the IHC-mediated transduction process shows a significant variability across individual synapses. The results of the simulations show that the average discharge rate of AFs exhibit a preference to low-frequency oscillations of the ciliary bundle and that this preference can be accurately described with the same function for all simulated AFs. The difference in the stereocilia deflection amplitude required to drive the different AFs above threshold at low $(\leq 500 \mathrm{~Hz})$ and high driving frequencies $(\geq 4 \mathrm{kHz})$ varies from 1.4 to $9.2 \mathrm{~dB}$, depending on the properties of the considered synapse.

The largest contributing factor to this variability is the offset of the synaptic transfer function, which controls the gating of the exocytosis at the synapses. Heterogeneity of the gating properties at the individual synapses is evident from recordings from afferent AFs: units with similar discharge rates at saturation can exhibit spontaneous discharge rates spanning from less than 0.1 to more than 100 spikes/s (Liberman, 1978). Diverse gating properties of the $\mathrm{Ca}^{2+}$ channels in the same IHC have been documented by Frank et al. (2009) and Ohn et al. (2016), who found a large variance in the half-activation potential and number of the $\mathrm{Ca}^{2+}$ channels across active zones in the same IHC. Due to the impossibility of estimating the precise relationship between $\mathrm{Ca}^{2+}$ signaling and exocytosis at the synapse, the slope and offset of the synaptic transfer function have been chosen in order to fit the peak and spontaneous rate of the afferent AF, given the macroscopic $\mathrm{Ca}^{2+}$ current.

Although the behavior of the simplified model employed in this study does not heavily depend on the activation of the $\mathrm{Ca}^{2+}$ channels relative to the receptor potential (Sec. III E), the model outcome strongly supports the notion that the gating and the number of the $\mathrm{Ca}^{2+}$ channels play a major role in the differentiation between low and high spontaneous rate fibers. In particular, the found variability in the parameters of the synaptic transfer function might account for the differences in the size and gating of the $\mathrm{Ca}^{2+}$ channel clusters among individual synapses.

For example, the assumption that the half-activation of the $\mathrm{Ca}^{2+}$ channels is $-30 \mathrm{mV}$ for synapses with the highest 
peak and spontaneous rate and of $-20 \mathrm{mV}$ for those with the lowest spontaneous and peak rate would lead to a 4.5 -fold variation in the slope of the synaptic transfer function $[z$ in Eq. (14)]. This variability in the slope of the synaptic transfer function is comparable to the differences between the maximal $\mathrm{Ca}^{2+}$ influx at the different synapses (Meyer et al., 2009; Frank et al., 2009), which is thought to arise from the difference in the number of $\mathrm{Ca}^{2+}$ channels in the different active zones (Ohn et al., 2016). Therefore, the variability in the slope of the synaptic transfer function necessary to relate the macroscopic $\mathrm{Ca}^{2+}$ current with the exocytosis rate might just reflect the differences in the slope of the activation of the $\mathrm{Ca}^{2+}$ current between different synapses near the IHC resting potential, which stem from the heterogeneity in the size and gating properties of the synaptic $\mathrm{Ca}^{2+}$ channels clusters.

The apparent offset in the synaptic transfer function that relates the whole-cell current with the exocytosis rate at a single synapse, which was necessary to fit the dynamic range of the AFs' discharge rate in the model, most likely stems from a non-perfectly linear relationship between the wholecell $\mathrm{Ca}^{2+}$ current and the exocytosis rate at individual synapses. Such nonlinearity might be caused by the high intrinsic $\mathrm{Ca}^{2+}$ cooperativity (Keen and Hudspeth, 2006) or from high channel cooperativity as Heil and Neubauer (2010) propose. In either of the cases, the mismatch between the $\mathrm{Ca}^{2+}$ influx at the considered synapse vs the average $\mathrm{Ca}^{2+}$ influx measured across all the synapses of the same IHC would lead to an apparent offset in the synaptic transfer function relating whole-cell current and exocytosis rate at a single synapse.

\section{CONCLUSION}

This paper presented a computational method to estimate the frequency tuning of the inner hair cell (IHC) stereocilia from neural tuning curves. To achieve this goal, a computational model of the mechanical-to-neural transduction was developed by including the equations describing the activation of the principal ion channels in the IHC. The model parameters are based on results of previously published in vitro measurements performed on mammalian IHCs. The predicted $\mathrm{Ca}^{2+}$ current at the synapse was employed to predict the $\mathrm{Ca}^{2+}$-driven rate of release of neurotransmitters at the synapse. Because the analysis focused on levels of excitation near the threshold of the afferent auditory fibers, the effects of refractoriness and synaptic depression were considered negligible in the determination of the neural tuning curves.

The model predicts that the vibrations of the stereocilia necessary to drive the afferent auditory fibers above threshold are smaller at low driving frequencies than at high driving frequencies. This difference is well described by a lowfrequency plateau exponentially decaying above about $500 \mathrm{~Hz}$. Depending on the response properties of the considered auditory fiber, there is a substantial heterogeneity in the level of the low-frequency plateau, spanning from 1.4 to $9.2 \mathrm{~dB}$. The predicted heterogeneity is thought to arise from the diversity of $\mathrm{Ca}^{2+}$ signaling at individual synapses, a well-documented phenomenon (Frank et al., 2009; Ohn et al., 2016).

Finally, the model predictions were employed to derive constant-stereocilia-deflection contours from previously published tuning curves that present the tuning of the basilar membrane and that of the auditory nerve from the same cochlea (Narayan et al., 1998). The estimated constant-stereocilia-deflection contours are more similar to constant-basilar-membrane-velocity contours than neural tuning curves, pointing out that the frequency tuning of the stereocilia resembles that of basilar membrane velocity for frequencies above $500 \mathrm{~Hz}$. For frequencies below $500 \mathrm{~Hz}$, there is an apparent difference between the tuning of the stereocilia and that of the basilar membrane. These results are in stark contrast with the classical view of the stereocilia being approximately driven by basilar membrane velocity at low frequencies and basilar membrane displacement at high frequencies.

In particular, the application of the proposed method to existing data supports the idea that no simple linear relationship between basilar membrane and stereocilia vibrations exists in the mammalian cochlea, even for vibrations of the basilar membrane in the order of $1 \mathrm{~nm}$. Despite the technical difficulties in observing the deflection of the stereocilia directly, the present study proposes that it can be deduced from auditory nerve recordings using computational tools.

\section{ACKNOWLEDGMENTS}

We thank Ray Meddis, Laurel Carney, Leo McCormack, and Janani Fernandez and two anonymous reviewers for many helpful comments. We also thank Luis Costa for proofreading the manuscript. This work was supported by the Academy of Finland, the Aalto ELEC doctoral school, and by the DFG SPP1608 VE924/1-1.

\footnotetext{
${ }^{1}$ The order of the activation of a population of ion channels corresponds to the order of the differential equation describing the evolution of the fraction of open channels.

${ }^{2}$ Kros and Crawford (1990) measured the activation time constants of the $\mathrm{K}^{+}$channels at room temperature and then estimated the values of such constants at body temperature assuming a Q10 factor of 3 .

${ }^{3}$ Heil and Neubauer (2010) argued that the apparent linear relationship between whole-cell $\mathrm{Ca}^{2+}$ current and whole-cell exocytosis rate can result from a cubic relationship between $\mathrm{Ca}^{2+}$ current and the exocytosis rate at individual synapses. Although their theory is hard to reconcile with the recordings from the synaptic bouton of the immature rat by Goutman and Glowatzki (2007), it is an open possibility. In any case, as we discuss in Secs. II B 2 and IV C, because the voltage-gating of the $\mathrm{Ca}^{2+}$ current differs among synapses (Frank et al., 2009; Ohn et al., 2016), it is very unlikely that a truly power-law relationship exists between whole-cell current and exocytosis rate at a single synapse. In this respect, the quasilinear function we adopt here might be thought of as the first-order expansion of a nonlinear function. This formulation might be suitable to approximate the relationship between whole-cell $\mathrm{Ca}^{2+}$ and the exocytosis rate in certain intervals even in the case a cubic relationship between the $\mathrm{Ca}^{2+}$ current and the exocytosis rate existed at individual synapses.

${ }^{4}$ The steady-state rates employed in the referenced study are the average discharge rates of AF responses to short tonal stimuli starting from 10 to $15 \mathrm{~ms}$ after the onset, where the AF responses have not really reached a steady-state discharge rate; the actual steady-state rates are of much lower values (Kiang et al., 1965).

${ }^{5}$ The cut-off frequency corresponding to the activation time constant of the MET channels is 2.5 octaves above that corresponding to the IHC membrane time constant and 2 octaves above that associated with the activation
} 
time constant of the $\mathrm{Ca}^{2+}$ channels. Therefore, the time course of the activation of the MET channels plays a very minor if not negligible role in shaping the frequency sensitivity of the AFs. This consideration is particularly important for the validity of the results drawn here, because the activation time constant of the MET channels might be shorter than that employed here (see Ricci et al., 2005).

${ }^{6}$ This estimation is correct if the displacement of the stereocilia increases linearly with the stimulus pressure. The application of the proposed method is appropriate for high CF units because the differences between neural and stereocilia frequency tuning are significant at frequencies well below $\mathrm{CF}$, where the mechanical responses grow approximately linearly (Robles and Ruggero, 2001).

Brandt, A., Khimich, D., and Moser, T. (2005). "Few Ca 1.3 channels regulate the exocytosis of a synaptic vesicle at the hair cell ribbon synapse," J. Neurosci. 25(50), 11577-11585.

Brandt, A., Striessnig, J., and Moser, T. (2003). " $\mathrm{Ca}_{\mathrm{V}} 1.3$ channels are essential for development and presynaptic activity of cochlear inner hair cells," J. Neurosci. 23(34), 10832-10840.

Cheatham, M., and Dallos, P. (1999). "Response phase: A view from the inner hair cell," J. Acoust. Soc. Am. 105(2), 799-810.

Chen, F., Zha, D., Fridberger, A., Zheng, J., Choudhury, N., Jacques, S. L., Wang, R. K., Shi, X., and Nuttall, A. L. (2011). "A differentially amplified motion in the ear for near-threshold sound detection," Nat. Neurosci. 14(6), 770-774.

Corns, L. F., Johnson, S. L., Kros, C. J., and Marcotti, W. (2014). "Calcium entry into stereocilia drives adaptation of the mechanoelectrical transducer current of mammalian cochlear hair cells," Proc. Natl. Acad. Sci. U.S.A. 111(41), 14918-14923.

Frank, T., Khimich, D., Neef, A., and Moser, T. (2009). "Mechanisms contributing to synaptic $\mathrm{Ca}^{2+}$ signals and their heterogeneity in hair cells," Proc. Natl. Acad. Sci. U.S.A. 106(11), 4483-4488.

Freeman, D. M., and Weiss, T. F. (1990). "Hydrodynamic forces on hair bundles at high frequencies," Hear. Res. 48(1-2), 31-36.

Goutman, J. D., and Glowatzki, E. (2007). "Time course and calcium dependence of transmitter release at a single ribbon synapse," Proc. Natl. Acad. Sci. U.S.A. 104(41), 16341-16346.

Guinan, J. J. (2012). "How are inner hair cells stimulated? Evidence for multiple mechanical drives," Hear. Res. 292(1), 35-50.

Heil, P., and Neubauer, H. (2010). "Summing across different active zones can explain the quasi-linear $\mathrm{Ca} 2+-$ dependencies of exocytosis by receptor cells," Front. Synaptic Neurosci. 2, 148

Howard, J., and Hudspeth, A. (1987). "Mechanical relaxation of the hair bundle mediates adaptation in mechanoelectrical transduction by the bullfrog's saccular hair cell,” Proc. Natl. Acad. Sci. U.S.A 84(9), 3064-3068.

Hudspeth, A., and Lewis, R. (1988). "Kinetic analysis of voltage- and iondependent conductances in saccular hair cells of the bull-frog, Rana catesbeiana," J. Physiol. 400(1), 237-274.

Jia, S., Dallos, P., and He, D. Z. (2007). "Mechanoelectric transduction of adult inner hair cells," J. Neurosci. 27(5), 1006-1014.

Johnson, S. L. (2015). "Membrane properties specialize mammalian inner hair cells for frequency or intensity encoding," Elife 4, e08177.

Johnson, S. L., Beurg, M., Marcotti, W., and Fettiplace, R. (2011). "Prestindriven cochlear amplification is not limited by the outer hair cell membrane time constant," Neuron 70(6), 1143-1154.

Johnson, S. L., Forge, A., Knipper, M., Münkner, S., and Marcotti, W. (2008). "Tonotopic variation in the calcium dependence of neurotransmitter release and vesicle pool replenishment at mammalian auditory ribbon synapses," J. Neurosci. 28(30), 7670-7678.

Johnson, S. L., and Marcotti, W. (2008). "Biophysical properties of $\mathrm{Ca}_{\mathrm{V}} 1.3$ calcium channels in gerbil inner hair cells," J. Physiol. 586(4), 1029-1042.

Johnson, S. L., Marcotti, W., and Kros, C. J. (2005). "Increase in efficiency and reduction in $\mathrm{Ca}^{2+}$ dependence of exocytosis during development of mouse inner hair cells," J. Physiol. 563(1), 177-191.

Karavitaki, K. D., and Mountain, D. C. (2007). "Evidence for outer hair cell driven oscillatory fluid flow in the tunnel of Corti," Biophys. J. 92(9), 3284-3293.

Keen, E. C., and Hudspeth, A. (2006). "Transfer characteristics of the hair cell's afferent synapse," Proc. Natl. Acad. Sci. U.S.A 103(14), 5537-5542.

Kennedy, H. J., Evans, M. G., Crawford, A. C., and Fettiplace, R. (2003). "Fast adaptation of mechanoelectrical transducer channels in mammalian cochlear hair cells," Nat. Neurosci. 6(8), 832-836.
Kiang, N. Y. S., Watanabe, T., Thomas, E. C., and Clark, L. F. (1965). Discharge Patterns of Single Fibers in the Cat's Auditory Nerve (MIT Press, Cambridge, MA).

Kros, C., and Crawford, A. (1990). "Potassium currents in inner hair cells isolated from the guinea-pig cochlea," J. Physiol. 421(1), 263-291.

Kros, C. J., Ruppersberg, J. P., and Rüsch, A. (1998). "Expression of a potassium current in inner hair cells during development of hearing in mice," Nature 394(6690), 281-284.

Kros, C., Rusch, A., and Richardson, G. (1992). "Mechano-electrical transducer currents in hair cells of the cultured neonatal mouse cochlea," Proc. R. Soc. B 249(1325), 185-193.

Lee, H. Y., Raphael, P. D., Park, J., Ellerbee, A. K., Applegate, B. E., and Oghalai, J. S. (2015). "Noninvasive in vivo imaging reveals differences between tectorial membrane and basilar membrane traveling waves in the mouse cochlea," Proc. Natl. Acad. Sci. U.S.A. 112(10), 3128-3133.

Liberman, M. C. (1978). "Auditory-nerve response from cats raised in a low-noise chamber,” J. Acoust. Soc. Am. 63(2), 442-455.

Lim, D. J. (1980). "Cochlear anatomy related to cochlear micromechanics. A review," J. Acoust. Soc. Am. 67(5), 1686-1695.

Lopez-Poveda, E. A., and Eustaquio-Martín, A. (2006). "A biophysical model of the inner hair cell: The contribution of potassium currents to peripheral auditory compression," JARO 7(3), 218-235.

Marcotti, W., Johnson, S. L., and Kros, C. J. (2004). "Effects of intracellular stores and extracellular $\mathrm{Ca}^{2+}$ on $\mathrm{Ca}^{2+}$-activated $\mathrm{k}+$ currents in mature mouse inner hair cells," J. Physiol. 557(2), 613-633.

Meyer, A. C., Frank, T., Khimich, D., Hoch, G., Riedel, D., Chapochnikov, N. M., Yarin, Y. M., Harke, B., Hell, S. W., Egner, A., and Moser, T. (2009). "Tuning of synapse number, structure and function in the cochlea," Nat. Neurosci. 12(4), 444-453.

Mountain, D. C., and Cody, A. R. (1999). "Multiple modes of inner hair cell stimulation," Hear. Res. 132(1), 1-14.

Narayan, S. S., Temchin, A. N., Recio, A., and Ruggero, M. A. (1998). "Frequency tuning of basilar membrane and auditory nerve fibers in the same cochleae," Science 282(5395), 1882-1884.

Nowotny, M., and Gummer, A. W. (2006). "Nanomechanics of the subtectorial space caused by electromechanics of cochlear outer hair cells," Proc. Natl. Acad. Sci. U.S.A. 103(7), 2120-2125.

Ohn, T.-L., Rutherford, M. A., Jing, Z., Jung, S., Duque-Afonso, C. J., Hoch, G., Picher, M. M., Scharinger, A., Strenzke, N., and Moser, T. (2016). "Hair cells use active zones with different voltage dependence of $\mathrm{Ca}^{2+}$ influx to decompose sounds into complementary neural codes," Proc. Natl. Acad. Sci. U.S.A. 113(32), E4716-E4725.

Palmer, A., and Russell, I. (1986). "Phase-locking in the cochlear nerve of the guinea-pig and its relation to the receptor potential of inner hair-cells," Hear. Res. 24(1), 1-15.

Peterson, A. J., Irvine, D. R., and Heil, P. (2014). "A model of synaptic vesicle-pool depletion and replenishment can account for the interspike interval distributions and nonrenewal properties of spontaneous spike trains of auditory-nerve fibers," J. Neurosci. 34(45), 15097-15109.

Platzer, J., Engel, J., Schrott-Fischer, A., Stephan, K., Bova, S., Chen, H., Zheng, H., and Striessnig, J. (2000). "Congenital deafness and sinoatrial node dysfunction in mice lacking class d l-type $\mathrm{Ca}^{2+}$ channels," Cell 102(1), 89-97.

Ramamoorthy, S., Zha, D., Chen, F., Jacques, S. L., Wang, R., Choudhury, N., Nuttall, A. L., and Fridberger, A. (2014). "Filtering of acoustic signals within the hearing organ," J. Neurosci. 34(27), 9051-9058.

Ren, T., He, W., and Barr-Gillespie, P. G. (2016a). "Reverse transduction measured in the living cochlea by low-coherence heterodyne interferometry," Nat. Commun. 7, 10282.

Ren, T., He, W., and Kemp, D. (2016b). "Reticular lamina and basilar membrane vibrations in living mouse cochleae," Proc. Natl. Acad. Sci. U.S.A 113(35), 9910-9915.

Rhode, W. S., and Smith, P. H. (1985). "Characteristics of tone-pip response patterns in relationship to spontaneous rate in cat auditory nerve fibers," Hear. Res. 18(2), 159-168.

Ricci, A. J., Kennedy, H. J., Crawford, A. C., and Fettiplace, R. (2005). "The transduction channel filter in auditory hair cells," J. Neurosci. 25(34), 7831-7839.

Ricci, A., Wu, Y., and Fettiplace, R. (1998). "The endogenous calcium buffer and the time course of transducer adaptation in auditory hair cells," J. Neurosci. 18(20), 8261-8277.

Robles, L., and Ruggero, M. A. (2001). "Mechanics of the mammalian cochlea," Physiol. Rev. 81(3), 1305-1352. 
Rutherford, M. A., Chapochnikov, N. M., and Moser, T. (2012). "Spike encoding of neurotransmitter release timing by spiral ganglion neurons of the cochlea," J. Neurosci. 32(14), 4773-4789.

Shamma, S. A., Chadwick, R. S., Wilbur, W. J., Morrish, K. A., and Rinzel, J. (1986). "A biophysical model of cochlear processing: Intensity dependence of pure tone responses," J. Acoust. Soc. Am. 80(1), 133-145.

Siegel, J. (1992). "Spontaneous synaptic potentials from afferent terminals in the guinea pig cochlea," Hear. Res. 59(1), 85-92.

Steele, C. R., Boutet de Monvel, J., and Puria, S. (2009). "A multiscale model of the organ of Corti," J. Mech. Mater. Struct. 4(4), 755-778.

Steele, C. R., and Puria, S. (2005). "Force on inner hair cell cilia," Int. J. Solids Struct. 42(21), 5887-5904.

Taberner, A. M., and Liberman, M. C. (2005). "Response properties of single auditory nerve fibers in the mouse," J. Neurophysiol. 93(1), 557-569.

Temchin, A. N., and Ruggero, M. A. (2010). "Phase-locked responses to tones of chinchilla auditory nerve fibers: Implications for apical cochlear mechanics," JARO 11(2), 297-318.

van Netten, S. M., Dinklo, T., Marcotti, W., and Kros, C. J. (2003). "Channel gating forces govern accuracy of mechano-electrical transduction in hair cells," Proc. Natl. Acad. Sci. U.S.A 100(26), 15510-15515.
Vannucci, G., and Teich, M. C. (1978). "Effects of rate variation on the counting statistics of dead-time-modified Poisson processes," Opt. Commun. 25(2), 267-272.

Winter, I. M., Robertson, D., and Yates, G. K. (1990). "Diversity of characteristic frequency rate-intensity functions in guinea pig auditory nerve fibres," Hear. Res. 45(3), 191-202.

Wong, A. B., Jing, Z., Rutherford, M. A., Frank, T., Strenzke, N., and Moser, T. (2013). "Concurrent maturation of inner hair cell synaptic $\mathrm{Ca}^{2+}$ influx and auditory nerve spontaneous activity around hearing onset in mice," J. Neurosci. 33(26), 10661-10666.

Wong, A. B., Rutherford, M. A., Gabrielaitis, M., Pangršič, T., Göttfert, F., Frank, T., Michanski, S., Hell, S., Wolf, F., Wichmann, C., and Moser, T. (2014). "Developmental refinement of hair cell synapses tightens the coupling of $\mathrm{Ca}^{2+}$ influx to exocytosis," EMBO J. 33(3), 247-264.

Zagaeski, M., Cody, A. R., Russell, I. J., and Mountain, D. C. (1994). "Transfer characteristic of the inner hair cell synapse: Steady-state analysis," J. Acoust. Soc. Am. 95(6), 3430-3434.

Zeddies, D. G., and Siegel, J. H. (2004). "A biophysical model of an inner hair cell," J. Acoust. Soc. Am. 116(1), 426-441.

Zetes, D. E., and Steele, C. R. (1997). "Fluid-structure interaction of the stereocilia bundle in relation to mechanotransduction," J. Acoust. Soc. Am. 101(6), 3593-3601. 